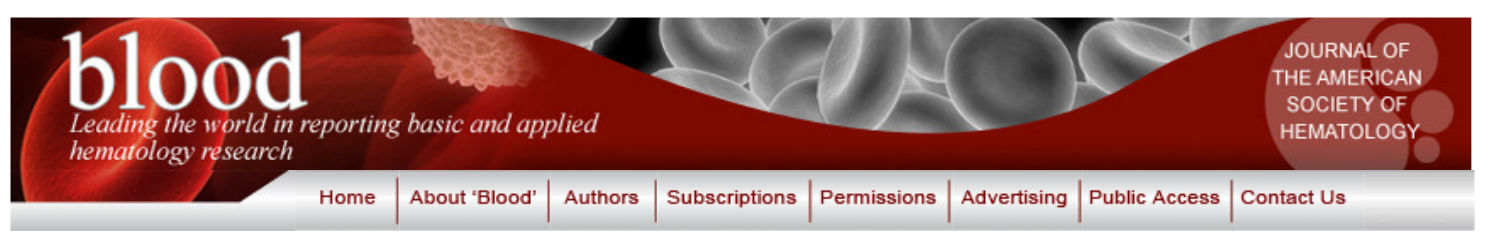

\begin{tabular}{l|}
\hline SEARCH: \\
\hline Advanced \\
\hline Current Issue \\
\hline First Edition \\
\hline e-Letters \\
\hline Future Articles \\
\hline Archives \\
\hline Submit to Blood \\
\hline Search Blood \\
\hline ASH \\
\hline Meeting Abstracts \\
\hline E-Mail Alerts
\end{tabular}

Blood (ASH Annual Meeting Abstracts) 2008 112: Abstract 5094 (1) 2008 American Society of Hematology

Publication Only

This Article

Myelodysplastic Syndromes

Services

\title{
Evaluation of Apoptotic Molecular Markers in Myelodysplastic Syndrome Patients
}

Emília Cortesão, 1,*, Ana Cristina Gonçalves ${ }^{2, *}$, Maria Isabel Sousa, , Carlos Moucho ${ }^{3, *}$, Luís Rito ${ }^{3, *}$, Emília Magalhães, ${ }^{3, *}$, Ana Isabel Espadana ${ }^{3, *}$, Maria Amélia Pereira ${ }^{4, *}$, Adriana Teixeira ${ }^{3, *}$, José Nascimento Costa ${ }^{1, *}$ and Ana Bela Sarmento ${ }^{2, *}$

Email this article to a friend Download to citation manager Citing Articles Citing Articles via Google Scholar Google Scholar Articles by Cortesão, $\mathrm{E}$. Articles by Sarmento, A. B. Search for Related Content PubMed

${ }^{1}$ University Hospital of Coimbra; Faculty of Medicine and CIMAGO, University of Coimbra, Coimbra, Portugal, ${ }^{2}$ Faculty of Medicine and CIMAGO, University of Coimbra, Coimbra, Portugal, ${ }^{3}$ University Hospital of Coimbra, Coimbra, Portugal, ${ }^{4}$ Distrital Hospital of Figueira da Foz, Figueira da Foz, Portugal

Abstract

Articles by Sarmento, A. B.

Social Bookmarking

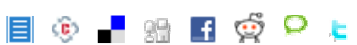

What's this?

The aetiology and pathogenesis of Myelodysplastic Syndrome (MDS) remain poorly characterized. One mechanism invoked to explain the apparent discrepancy between cellular marrow and peripheral blood cytopenias in patients with MDS is programmed cell death (apoptosis), which occurs with increased frequency in MDS marrow. Higher rates of apoptosis have been observed in Refractory Anaemia (RA) and Refractory Anaemia with Excess Blasts (RAEB) compared with transforming MDS or acute leukaemia while others have found increased rates in late stages of disease. Cell death may be triggered by activated $\mathrm{T}$ cells (in an attempt to eliminate the armful clone), secretion of death proteins/inhibitory cytokines ( $\mathrm{TNF}_{\mathrm{x}}$, Fas/Fas-L) and interleukin-6, expression of the pro-apoptotic proteins of Bcl-2 family, and/or through a relative deficiency in hematopoietic growth factors (stromal defects). Increased expression and release of TNF-related apoptosis inducing ligand (TRAIL), also known as Apo2 ligand (Apo2L) at the bone marrow level is likely to impair erythropoiesis and to contribute to the degree of anaemia, the major clinical feature of MDS. The exact mechanism by which TRAIL eliminates tumour cells preferentially or selectively is not known. One possibility is differential expression of agonistic receptors 1 and 2 (TRAIL-R1 and -R2), also known as death receptors, DR4 and DR5, and antagonistic or modulatory receptors, TRAIL-R3 and R4, also known as decoy receptors, DcR1 and DcR2. An alternative mechanism would be differential expression of, or functional defects in, cytoplasmatic inhibitors of apoptosis such as FADD-like converting enzyme (ICE)inhibitory protein (FLIP) or other inhibitory IL -1 apoptotic proteins (IAP) likely survivin. Although increased programmed cell death in MDS may occur as an early rescue or compensatory response to deregulated clonal proliferation, it may also represent a pathophysiologic consequence of the epigenetic changes associated with the disease biology of MDS as we have already referred (EHA 2008). However the MDS remain an inadequately characterized disorder and novel strategies for studying disease pathogenesis and progression continue to shape our understanding and approach to diagnosing and treating. With this study we hope to contribute to the identification of molecular markers involved in apoptotic signalling pathways that could be related with MDS and that could interfere with the prognosis, namely the evolution to acute leukaemia, and with the therapeutic approach. For this purpose we have examined the expression of proteins involved in apoptotic pathways (membranar and mitochondrial) namely Bax, Bcl-2, p53, Fas/FasL, TRAIL/TRAIL-Rs and survivin, in CD34 bone marrow cells populations collected at diagnosis from 23 patients with de novo MDS and in 6 ITP (non-malignant controls). The expression of the proteins was evaluated by flow cytometry using monoclonal antibodies. The median age was 77 years (33-84), $\mathrm{M} / \mathrm{F}=12 / 11$, WHO subtypes: RCMD ( $\mathrm{n}=12)$, RA ( $\mathrm{n}=2)$, RCMD-RS ( $\mathrm{n}=1)$, CMML ( $\mathrm{n}=2)$, RAEB-2 $(\mathrm{n}=5), 5 \mathrm{q}-$ syndrome $(n=1)$ and IPSS: low $(n=6)$, intermediate- $1(n=6)$ and intermediate- $2(n=2)$, in patients with cytogenetic results. Two of the patients (both RAEB-2) evolve to acute leukemia, with a median follow-up of 24 months (12-65). Our results show that CD34 MDS patients cells have an higher Bax/Bcl-2 ratio, Bax, survivin, cit c, TRAIL-R3 and TRAIL-R4 levels and lower levels of Bcl-2, TRAIL, TRAIL-R1, Fas and FasL compared with non-malignant controls. However RA patients have the higher Bax, Fas, Fas-L, TRAIL-R3 and survivin levels while RAEB-2 patients have the lowest. This subtype of patients (RAEB-2) also show a decrease in Bax/Bcl-2 ratio, lower levels of TRAIL-R2 and higher levels of Bcl-2. This study suggest that apoptosis deregulation may be implicated in MDS. However, in RA an increase in apoptosis predominates while in RAEB the resistance to apoptosis may contribute to a less favourable prognosis and may influence the transition to acute myeloid leukaemia. Furthermore, we hope that the identification of all that molecular markers may contribute to identify new risk patients groups and molecular therapeutic targets. 
Evaluation of Apoptotic Molecular Markers in Myelodysplastic Syndrome Patients -- ... Page 2 of 2

\section{Footnotes}

Corresponding author

Disclosures: No relevant conflicts of interest to declare.

Work supported by Center of Investigation in Environment Genetics and Oncobiology(CIMAGO). First and second authors - equal contribution on this study.

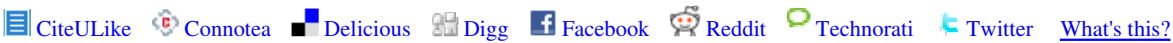

Click for information regarding free online access to various full-text Blood articles

Home About 'Blood' Authors Subscriptions Permissions Advertising Public Access Contact Us

Copyright $\odot 2008$ by American Society of Hematology $\quad$ Online ISSN: 1528-0020

http://abstracts.hematologylibrary.org/cgi/content/abstract/112/11/5094?sid=a59c6a5... 17-11-2011 